\title{
Stemness and Epithelial-Mesenchymal Transition of Breast Cancer Cells Incubated on Viscoelastic Gel Substrates
}

\author{
Ryohei Онта and Masami Окамото ${ }^{\dagger}$ \\ Department of Advanced Science and Technology, Graduate School of Engineering, Toyota Technological Institute, \\ 2-12-1 Hisakata, Tempaku, Nagoya 468-8511, Japan \\ (Received : January 26, 2021)
}

\begin{abstract}
Acrylamide copolymer-based gel substrates with different viscoelasticity were employed to evaluate the viscoelasticity effect on the direct relation among cancer stemness, cellular motility and mesenchymal properties with induction of epithelial-mesenchymal transition (EMT) of human breast adenocarcinoma (MCF-7) cells in both normoxia and hypoxia. Cellular migration speed $(S)$ of MCF-7 cells was significantly upregulated with decreasing in coefficient of damping $(\tan \delta)$. The softer gel substrate produced a large amount of surface molecule of cancer stem cells (CSC) marker CD44. In contrast, for the stem cell biomarker CD133 expression, their $\tan \delta$-dependent manner was not contributed by EMT phenomenon and was an independent from acquisition of the EMT. The substrate damping as potential physical parameter emerged the important linkage to cellular motility, cancer stemness, and EMT induction.
\end{abstract}

Key Words: Viscoelastic gel substrates / Epithelial-mesenchymal transition / Cancer stem cells

\section{INTRODUCTION}

Breast cancer is one of the most common malignancies in women and is the higher incidence and mortality rates among all female malignant tumors in $\mathrm{Japan}^{1-3)}$. The average 5 -year overall survival rate of breast cancer is reported to be around $55 \%$ due to the poor outcome of the therapy for metastatic disease in the resistance to radiation and chemothera$\mathrm{py}^{4)}$. The breast cancer therapies having poor prognosis prompt us to study an effective cancer therapy. That is, breakthroughs in effective cancer therapies are imperative.

A recently proposed hypothesis suggests that cancer stem cells (CSCs) and epithelial-mesenchymal transition (EMT) play a pivotal role in cancer metastasis, recurrence and drug resistance ${ }^{5}$. CSCs were first proposed by Virchow and Conheim; a subpopulation of cancer cells resembles the same traits as embryonic cells such as the ability to proliferate, and cancer is derived from the activation of dormant cells of the same tissue. A few CSCs do self-renew and produce a large number of heterogeneous and highly proliferative cancer cells to form primary tumors ${ }^{6}$.

EMT causes morphological and functional changes in epithelial cells, resulting in the acquisition of mesenchymal cell-like features by cancer cells, where an epigenic program

\footnotetext{
$\dagger$ Corresponding author.

E-mail : okamoto@toyota-ti.ac.jp (M. Okamoto)

Tel: +81-52-809-1861
}

leads epithelial cells to lose their cell-cell and cell-extracellular matrix (ECM) interactions to undergo cytoskeleton reorganization and to gain morphological and functional characteristics of mesenchymal cells. The tumor microenvironment can elicit EMT. The capability of metastatic cancers to shift motility modes by EMT is one of the main features of invasion. The understanding the interaction between microenvironment and cancer cells is also critical subject to progress in the cancer treatment ${ }^{7,8)}$.

Most proliferative cancer cells are killed by chemoradiotherapy. However, similar to sarcomas, CSCs undergoing EMT, which present mesenchymal features, are resistant to anti-cancer therapies. Thus, CSCs survive and cause cancer recurrence. Recent studies showed that inducible factors of EMT not only induce EMT, but also enhance CSC features of cancer cells ${ }^{9}$. These findings suggest that EMT is closely related to cancer progression.

Targeting the population of CSCs and EMT may result in breakthrough in cancer treatments. The connection between CSCs and EMT has attracted considerable attention. The induction of EMT is believed to promote CSC features. However, there is a lack of more detailed analysis on the role of EMT in modulating the stemness of breast cancer cells.

To solve this profound subject, artificial ECM with different viscoelastic properties is required which mimics in vivo microenvironment. This study was aimed at examining 
the effect of viscoelasticity of the substrate on the direct relation among cancer stemness, cellular motility and mesenchymal properties with induction of EMT in both normoxia and hypoxia $^{10)}$.

\section{MATERIALS AND METHODS}

\subsection{Preparation of acrylamide copolymer gels}

Acrylamide (AAm, Sigma-Aldrich), N-acryloyl-6aminocaproicacid (ACA, Santa Cruz), N,N'Methylenebisacrylamide (BIS, Sigma-Aldrich), ammonium persulfate (APS, Sigma-Aldrich), and $N, N, N^{\prime}, N^{\prime}-$ tetramethylethylendiamine (TEMED, Sigma-Aldrich), were used without further purification. Millipore Milli Q ultrapure (specific resistance: $18 \mathrm{M} \Omega \mathrm{cm}$, total organic carbon (TOC) $<20$ ppb, Merck Millipore Japan Co.) water through dialysis membrane was used in all experiment.

After polymerization, the AAm-ACA copolymer (AC) gels were fully hydrated (swelling) in phosphate-buffer saline (PBS, Nacalai Tesque, Kyoto) and washed with $0.1 \mathrm{M}$ of 2-(N-morpholino) ethanesulfonic acid buffer (MES, Dojindo) with $0.5 \mathrm{M} \mathrm{NaCl}$ (Sigma-Aldrich) at $\mathrm{pH} 6.1$. The details of preparation $^{11)}$ and rheological characterization ${ }^{12)}$ were described in previous papers.

\subsection{Immobilization of collagen on AC gels}

For conjugation of type-I collagen on the AC gel surface, the solution of $0.2 \mathrm{M}$ of 1-ethyl-3-(3-dimethyl aminopropyl) carbodiimide hydrochloride (EDAC, Tokyo Chemical Industry Co., Ltd., Japan (TCI)) and $0.5 \mathrm{M}$ of $N$-hydroxysuccinimide (NHS, TCI) was prepared in MES buffer and then dehydration condensation reaction was performed for $30 \mathrm{~min}$ at ambient temperature. The gels modified with EDAC were washed with cold $40 \%$ methanol diluted with PBS and subsequently reacted with type-I collagen (Cellmatrix I-C, Nitta Gelatin, Inc.) for overnight at $4{ }^{\circ} \mathrm{C}$ in HEPES (2-[4-(2-hydroxyethyl)piperazin-1-yl]ethane-sulfonic acid, Gibco, Life Technology) buffered saline solution (2 × HBS: $\mathrm{NaCl}, 280 \mathrm{mM}: \mathrm{Na}_{2} \mathrm{HPO}_{4}, 1.5 \mathrm{mM}$ : HEPES, $50 \mathrm{mM}$ ) to adjust at $\mathrm{pH} 9.0$. The AC gels were washed once with HEPES buffer at $4{ }^{\circ} \mathrm{C}$ and then washed three times with PBS at room temperature. Then AC gels modified with type-I collagen were sterilized with germicidal UV light for $30 \mathrm{~min}$. Finally, all AC gels were equilibrated in high glucose Dulbecco's modified Eagle's medium (DMEM) (Nacalai Tesque, Kyoto) supplemented with $10 \%(\mathrm{v} / \mathrm{v}) \mathrm{FBS}$ at $4{ }^{\circ} \mathrm{C}$ for overnight. Jiang et al. reported the resulting nanostructured collagen matrix was about $3 \mathrm{~nm}$ in thickness ${ }^{12)}$. For comparison, type-I collagen coated plates (TCP-coat) were prepared by adding Cellmatrix I-C solution onto 24-well tissue culture plates (TCP) and heated at $37{ }^{\circ} \mathrm{C}$ for $2 \mathrm{~h}$. Then added solution was removed. The details of preparation and characterization were described in our previous paper ${ }^{12}$.

\subsection{Cell culture}

Human breast adenocarcinoma cell line, MCF-7 (ATCC) were cultured in high glucose Dulbecco's modified Eagle's medium (DMEM) (Nacalai Tesque, Japan) supplemented with $10 \%$ (v/v) FBS, 100 unit/mL penicillin (Nacalai Tesque, Kyoto), and $100 \mu \mathrm{g} / \mathrm{mL}$ streptomycin (Nacalai Tesque, Kyoto), grown at $37^{\circ} \mathrm{C}$ under $5 \% \mathrm{CO}_{2}$ atmosphere and $95 \%$ relative humidity (normoxia) or hypoxic condition $\left(94 \% \mathrm{~N}_{2}\right.$, $5 \% \mathrm{CO}_{2}$, and $1 \% \mathrm{O}_{2}$ ) at $37^{\circ} \mathrm{C}$. Cells were grown to $70-80 \%$ confluence at normal culture condition. Cells were digested with trypsin and diluted to $5 \times 10^{3}$ cells $\mathrm{cm}^{-2}$ before being seeded onto the gel substrates.

\subsection{Real time polymerase chain reaction (RT- PCR)}

The total RNA was extracted from the cells cultured for three days using Fast Gene ${ }^{\mathrm{TM}}$ RNA Premium Kit (Nippon genetics). Then the RNA was subjected to reverse transcription using a Transcriptor Universal cDNA Master (Roche) following the manufacturer's instructions. The reaction solutions included $5 \mu \mathrm{L}$ of KAPA SYBR ${ }^{\circledR}$ FAST qPCR Master Mix (KAPA BIOSYSTEMS), $200 \mathrm{nM}$ forward and reverse predesigned primers ${ }^{13)}$, and $1 / 10$ of cDNA template in a $10 \mu \mathrm{L}$ volume. The resulting cDNA yield was then subjected to RT-PCR using Light Cycler $^{\circledR} 96$ system (Roche). The results were analyzed with Light Cycler ${ }^{\circledR} 96$ software (Roche). The cDNA samples were analyzed for expression of E-cadherin (CDH1), $\mathrm{N}$-cadherin (CDH2), transforming growth factor beta (TGF- $\beta$ ), hypoxia-inducible factor $1 \alpha$ (HIF-1 $\alpha$ ) and vimentin, relative to the glyceraldehyde-3-phosphatase dehydrogenase (GAPDH) as an internal standard for sample normalization. In addition, the cDNA samples of day 7 were analyzed for expression of snail family zinc finger 2 (SNAI2) and zinc finger E-box binding homeobox 1 (ZEB1). The details of characterization were described in our previous paper ${ }^{12)}$.

\subsection{Cellular migration}

MCF-7 cells were seeded on the gel substrates and cultured under normoxic or hypoxic conditions. Both cells were seeded at the density of $5 \times 10^{3}$ cells $\mathrm{cm}^{-2}$ for 3 days, pre-cultured for $24 \mathrm{~h}$ to attach the cells to the substrates. An environment of $37^{\circ} \mathrm{C}$ temperature, normoxic or hypoxic condition 
was applied during the time-lapse measurement. The cells were stained with $0.1 \mu \mathrm{g} \mathrm{mL} \mathrm{m}^{-1}$ of Hoechst 33342 for $20 \mathrm{~min}$ prior to time-lapse experiment. The blue fluorescent images of the tracers were obtained every 15 min for 16 hours by EVOS FL Auto fluorescent microscope (Life Technologies). To track the cell movement, the position of 20 individual centroids $[x(t), y(t)]$ of cell nucleus were obtained by a Mosaic particle tracker for ImageJ developed by Helmuth et al. ${ }^{14)}$. The start points of each cell were defined at the same position.

\subsubsection{Characterizing the cellular migration using the per-} sistent random walk (PRW) model

The obtained $x, y$ values were first transferred into mean squared displacement (MSD) as equation (1)

$$
\operatorname{MSD}(\tau)=\left\lfloor\left\langle(x(t+\tau)-x(t))^{2}+(y(t+\tau)-y(t))^{2}\right\rangle\right]_{n}
$$

where $x$ and $y$ are position of cells, $t$ is time, and $\tau$ is the time lag between positions of cells, $n$ is the number of tracked cells, $<\ldots>$ indicates time average, and [...] indicates cell average. Calculated MSD was assessed by applying model reported by several researchers ${ }^{15,16)}$. 2D MSD for PRW model can be expressed by equation (2)

$$
\operatorname{MSD}(\tau)=2 S^{2} P^{2}\left(e^{-\frac{\tau}{P}}+\frac{\tau}{P}-1\right)
$$

where $S$ is the cellular migration speed and $P$ is the persistent time. Cellular diffusivity $D$ is calculated as $D=S^{2} P / 2$.

\subsection{Flow cytometric analysis}

A total of $1 \times 10^{6}$ cells seeded onto the gel substrates for 7 days were suspended in $100 \mu \mathrm{L}$ PBS with $0.5 \%$ bovine serum albumin (BSA). The mouse anti-human CD133 mAb (Alexa Fluor 488-conjugated, Cell Signaling Technology) and rat anti-human $\mathrm{CD} 44 \mathrm{mAb}$ (PerCP-Cy5.5-conjugated, Cell Signaling Technology) were appropriately diluted in the FcR blocking reagent to a final volume of $20 \mu \mathrm{L}$. The antibodies were added to the cells, and mixture was incubated on ice according to the manufacturer's procedures. The cells were washed and resuspended in a suitable amount of buffer for analysis by flow cytometer (Attune ${ }^{\circledR}$ NxT Acoustic Focusing Cytometer, Thermo Fisher Scientific). Propidium iodide (Sigma-Aldrich) staining was applied to exclude the dead cells. Data analysis was performed with FlowJo v.10.0 Software.

\subsection{Statistics}

All data presented are expressed as the mean and standard deviations $( \pm \mathrm{SD})$. Statistical analysis was performed using one-way analysis of variance with Tukey-Kramer' post-hoc testing using Excel 2013 with Statcel4 v.2.0 Software (OMS Publishing Co., Tokyo, Japan), and significance was considered at a probability of $p<0.05$.

\section{RESULTS}

\subsection{Viscoelasticity of AC gels}

The viscoelastic features of the substrates (AC gel and TCP) are characterized by tan $\delta$ and storage modulus $\left(G^{\prime}\right)$ (see Fig. 1 in Ref. 12). The solid line indicates a viscoelastic figure of merit $(\mathrm{VFOM})\left(G^{\prime} / \rho\right) \times(\tan \delta)^{0.5}=10^{5} \mathrm{~m}^{2} \mathrm{~s}^{-2}$, with $\rho$ as density. With $\tan \delta$ values of $0.04-0.26$, VFOM values of AC gels $\left(\sim 10^{-2} \mathrm{~m}^{2} \mathrm{~s}^{-2}\right)$ are seven orders of magnitude lower than that of solid materials. This is achieved by soft matrix with cross-linker BIS molecule.

\subsection{Linkage to substrate viscoelasticity and mo- tility of cells}

Cellular migration speed $(S)$ in MCF-7 cells under hypoxia is upregulated with decreasing in damping coefficient $(\tan \delta)($ Fig. 1(d)). Although the stiff substrate increased cellular motility our results found that the migration speed is not robustly correlated with substrate stiffness (relative storage modulus $\left(G^{\prime} / \rho\right)$ : data not shown). This result suggests that the decreasing in damping is the driving force of the cellular migration in the cells. In addition, interestingly, the decreasing in $\tan \delta$ promotes the persistent time $(P)$ in a semi-log linear relation (Fig. 1(b, e)), while the results of the cellular diffusivity $(D)$ has no robust correlation with $\tan \delta$ (Fig. 1(c, f)).

\subsection{AC gel substrate contribution to CD133 and CD44 expression}

A subpopulation of cancer cells, the CSCs, was found to display stem cell characteristics that influence tumorigenesis. These CSCs have various cancer-promoting characteristics such as self-renewal differentiation, chemoresistance, and metastatic potential ${ }^{17,18)}$. CSCs express various CSC markers such as CD133, CD44, and CXCR4 ${ }^{19}$. The expression of CD133 is a strong predictor of declining prognosis, as high CD133 levels conversely relate to low 5-year overall survival and disease free survival rate in cancer patients ${ }^{20)}$. Another putative CSC marker is the cell-surface glycoprotein CD44, which was reported to be an adhesion molecule expressed in $\mathrm{CSCs}^{21)}$. When upregulated, CD44 increases tumor growth 
Normoxia
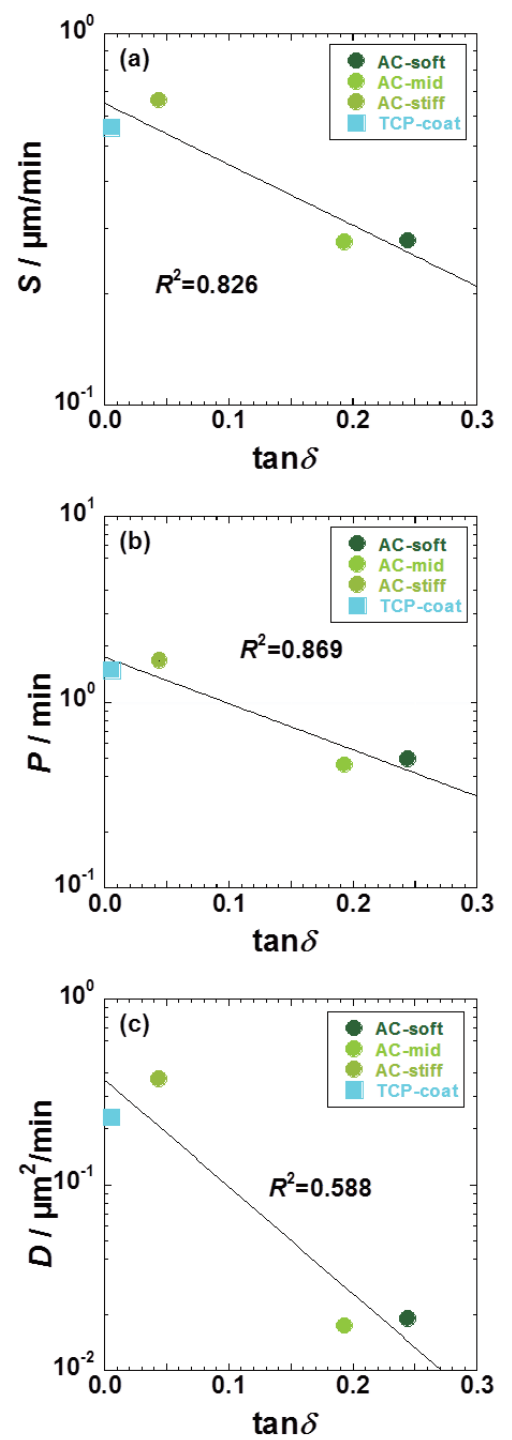

Hypoxia
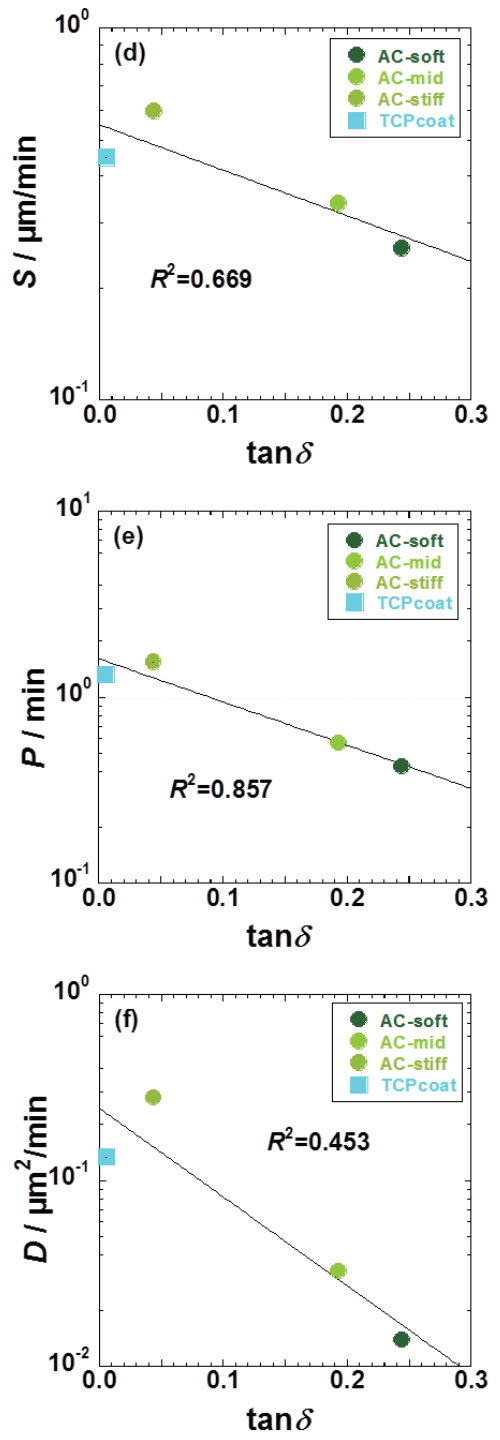

Fig. 1 Relationships between cellular migration speed $(S)((\mathrm{a})$ and $(\mathrm{d}))$, persistent time $(P)((\mathrm{b})$ and (e)), and cellular diffusivity $(D)((\mathrm{c})$ and $(\mathrm{f}))$ against damping coefficient $(\tan \delta)$ for MCF-7 cells at day 3 cultured on four different substrates under normoxic (a-c) and hypoxic (d-f) conditions. The solid line in each panel indicates semi-log linear regression.

and anti-apoptotic property ${ }^{22)}$. Both CD133 and CD44 are well-recognized stem cell biomarkers express in breast can$\operatorname{cer}^{23)}$.

To delineate CD133 and CD44 expression in MCF-7 cells incubated on six different AC gel substrates and TCPcoat, we used flow cytometry to analyze the expression of these surface markers for CSCs after double-staining with anti-CD133-FITC and anti-CD44-PreCP antibodies. Fig. 2 shows a distribution of CD133 and CD44 expression with a $\mathrm{CD}_{133}{ }^{+} \mathrm{CD} 44^{-}$population of $1.46 \%$, a $\mathrm{CD} 133^{-} \mathrm{CD} 44^{+}$population of $0.81 \%$, and a $\mathrm{CD} 133{ }^{+} \mathrm{CD} 44^{+}$population of $0.025 \%$ after the cells incubation on AC-soft (see Fig. 1 in Ref. 12) substrate in hypoxia at day 7. The multiple-marker detection (co-expression) exhibits very low percentage of population in the cells.
For comparison, the expression levels at each substrate tested in this study are plotted as a function of $\tan \delta$ (Fig. 3). The $\mathrm{CD}_{133}{ }^{+} \mathrm{CD} 44^{-}$levels are markedly elevated with increasing in $\tan \delta$ up to around 0.09 and have a decreasing trend for the cells cultured on AC-soft substrate under both oxygen concentration conditions (Fig. 3(a)).

Under normoxic and hypoxic conditions, the CD133CD $44^{+}$levels are promoted by damping coefficient. The level of $\mathrm{CD} 133^{-} \mathrm{CD} 44^{+}$is over 250 -fold for the cells cultured on AC-soft substrate in comparison with that on AC-s/o in normoxia, suggesting that the softer gel substrate produce a large amount of surface molecule of CD44 (Fig. 3(b)). From these findings, the expression of $\mathrm{CD} 133^{-} \mathrm{CD} 44^{+}$is uncoupled from that of $\mathrm{CD} 133^{+} \mathrm{CD} 44^{-}$in MCF-7 cells under different oxygen concentration levels. For the co-expression of 


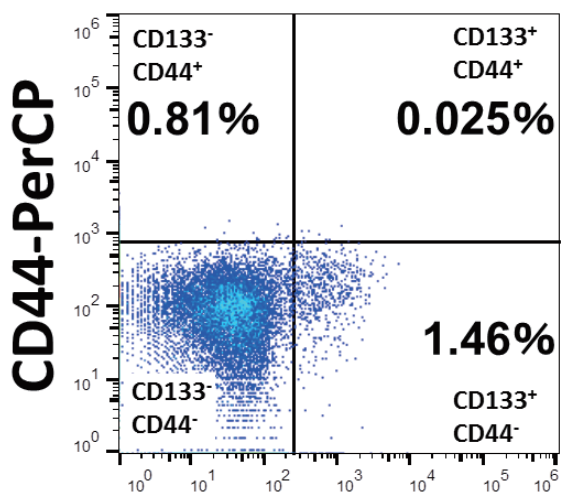

CD133-FITC

Fig. 2 Flow cytometry analysis of CD44 (y-axis) and CD133 (x-axis) -labeled MCF-7 cells for 7 days incubated on AC-soft in hypoxia. Dual staining of cells enable categorized of cells into four regions. The quadrants comprising each population are defined as $\mathrm{CD} 133^{-} \mathrm{CD} 44^{+}$,

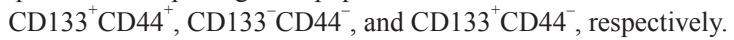
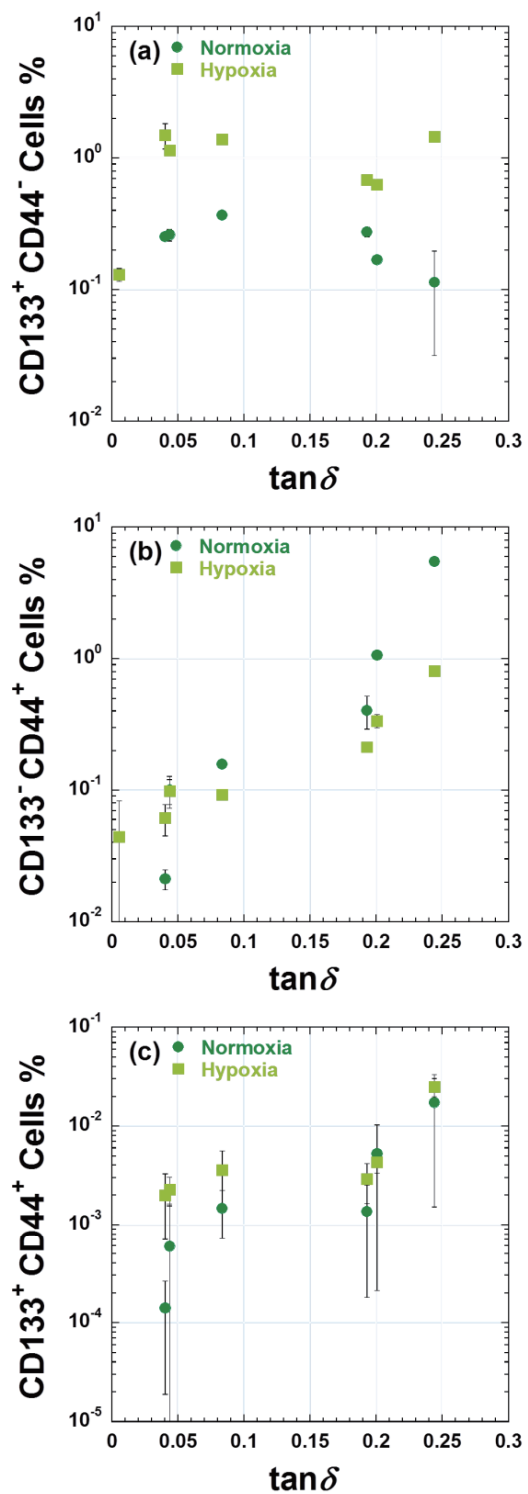

Fig. 3 Relationships between $\mathrm{CD} 133^{+} \mathrm{CD} 44^{-}$(a), $\mathrm{CD} 133^{-} \mathrm{CD} 44^{+}$(b), and $\mathrm{CD} 133^{+} \mathrm{CD} 44^{+}$(c) expression and damping coefficient $(\tan \delta)$ for MCF-7 cells at day 7 cultured on six different AC gel substrates and TCP-coat under both normoxic and hypoxic conditions.
$\mathrm{CD}_{133}{ }^{+} \mathrm{CD} 44^{+}$, more stem-like properties as compared with other population, seems to be affected by the combination of both $\mathrm{CD} 133^{+} \mathrm{CD} 44^{-}$and $\mathrm{CD} 133^{-} \mathrm{CD} 44^{+}$, i.e., a further increase of the levels in the cells incubated on AC-soft $(\tan \delta=0.244)$ via $\mathrm{AC}-\mathrm{mid}(\tan \delta=0.193)$ substrate is observed (Fig. 3(c)).

\subsection{AC gel substrate induced EMT}

The gene expression changes are investigated to understand the role of ECM for malignant phonotype. In this study, typical epithelial cell marker of E-cadherin (CDH1) and mesenchymal marker of vimentin and $\mathrm{CDH} 2^{24)}$ were chosen because EMT is a critical phenomenon induces cancer metasta$\operatorname{sis}^{25)}$.

To know detail of EMT and metastasis, transforming growth factor $\beta$ (TGF- $\beta)^{24)}$ and snail family zinc finger 2 $(\mathrm{SNAI} 2)^{24)}$ and zinc finger E-box binding homeobox 1 $(\mathrm{ZEB} 1)^{26)}$ were added to analysis. TGF- $\beta$ is known to induce EMT. SNAI2 and ZEB1 are potent repressor of epithelial cell marker E-cadherin (CHD1) gene expression. Cancer cells respond to the hypoxic microenvironment through the activity of hypoxia inducible factor $1 \alpha(\mathrm{HIF}-1 \alpha)^{27)}$ that behaves as a promotor of EMT.

Figure 4 shows the gene expression (HIF-1 $\alpha$, TGF- $\beta$, vimentin, $\mathrm{CDH} 2$, ZEB1, SNAI2, and $\mathrm{CDH} 1)$ for $\mathrm{MCF}-7$ cells cultured on AC gel substrates and TCP-coat (including TCP) at day 7 as a function of $\tan \delta$ under both normaxic and hypoxic environment.

In both normoxia and hypoxia, MCF-7 cells incubated on AC-soft $(\tan \delta=0.244)$ and AC-mid $(\tan \delta=0.195)$ substrates show a significant change in HIF-1 $\alpha$ expression (Fig. 4(a)). While significant repression is observed for the cells culture on AC-s/m $(\tan \delta=0.201)$. For TGF- $\beta$ level under hypoxic condition, similar change is obtained in MCF-7 cells on all AC gel substrates and TCP-coat (Fig. 4(b)). The cells incubated in the normoxic group do not show a significant increase in TGF- $\beta$ expression.

For vimentin expression, this surface protein is expressed with similar manner on AC gel substrates and TCPcoat (Fig. 4(c)). The vimentin level under hypoxic condition is markedly increased in the cells incubated on AC-soft (720fold) and AC-mid (360-fold) in comparison with that of incubation from AC-stiff substrate up to TCP-coat. Similar result is obtained when the cells are incubated in normoxia, while the cells express less vimentin (30-fold) in comparison with AC-soft and AC-stiff substrates.

The expression of CDH2 shows in Fig. 4(d). For CDH2 expression, similar changes (440-fold in hypoxia) are 

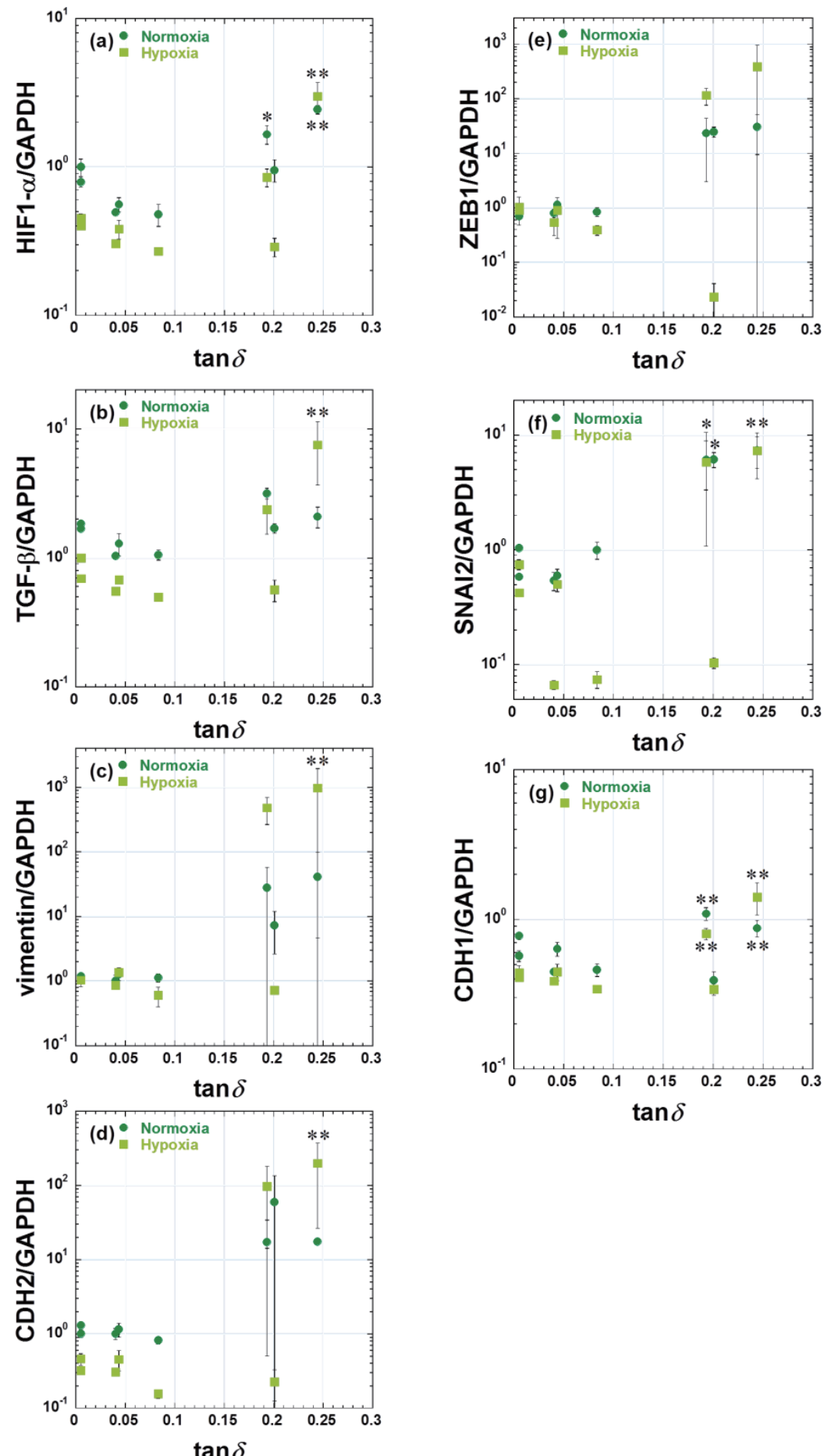

$\tan \delta$

Fig. 4 Effect of the substrate $\tan \delta$ on gene expression of (a) HIF-1 $\alpha$, (b) TGF- $\beta$, (c) Vimentin, (d) CDH2, (e) ZEB1 (f) SNAI2, and (g) CDH2 for MCF-7 cells after 7 days culture under both normoxia and hypoxia. $\left({ }^{*} p<0.05\right.$ and $\left.{ }^{* *} p<0.01\right)$.

obtained in the cells on AC gel substrates as compared with the results in vimentin expression (Fig. 4(c)). For transcription factors (ZEB1 (Fig. 4(e)) and SNAI2 (Fig. 4(f))), more upregulation of ZEB1 and SNAI2 in the cells incubated on AC-soft and AC-mid substrates in hypoxia has shown a trend to further induce EMT. The behavior is consistent with the results obtained in TGF- $\beta$ expression under both normoxic and hypoxic conditions.

As expected, the expression level of epithelial cell marker CHD1 associated with EMT is significantly lower than that observed in CDH2 expression level (Fig. 4(g)), indicating that EMT is more promoted in both normoxic and hypoxic environment.

Taken together, these results indicate that AC-soft and AC-mid substrates cause significant change in induction (transcription factors (ZEB1 and SNAI2) associated with EMT) and acquisition (vimentin and $\mathrm{CDH} 2$ expressions) of the EMT. Up to now, we have limited information regarding 
viscoelastic gel substrate-mediated EMT. The effect of $\tan \delta$ feature from AC-soft (0.244) to AC-mid (0.195) on gene expression might explain by a further study.

\section{DISCUSSION}

In each of gel substrates tested in this study, the effect of $\tan \delta$ feature on the gene expression of vimentin and $\mathrm{CDH} 2$ is more beneficial for MCF-7 cells under different oxygen concentration levels, in which a significant level of upregulation is evident for the cells incubated on AC-soft substrate.

When we correlate expression of $\mathrm{CD} 133^{-} \mathrm{CD} 44^{+}$with vimentin and/or CDH2 in MCF-7 cells, we observe a significant association between $\mathrm{CD} 133^{-} \mathrm{CD} 44^{+}$level and vimentin/ $\mathrm{CDH} 2$ expression in the cells incubated on AC-soft substrate. These results indicate that $\mathrm{CD} 133^{-} \mathrm{CD} 44^{+}$level is involved in regulating the expression of HIF-1 $\alpha$. We assume that breast CSCs characterized CD133ㄷำ $44^{+}$level may have a survival advantage under hypoxic condition, since EMT has been demonstrated to contribute to drug resistance in breast can$\operatorname{cer}^{28,29)}$.

So far, the induction of EMT is believed to promote CSC features. CSC theory has been widely accepted as a central principle to explain tumor aggressiveness, recurrence, chemoresistance and even metastasis through EMT phenomenon $^{9,28,30)}$.

The role of CD44, a hyaluronic receptor, is to promote cell-adhesion and assembly of cell surface growth factors, especially in the maintenance of cell-matrix interactions and maintenance of a stem cell phenotype ${ }^{31)}$. Mechanisms regarding how tan $\delta$ feature controls CD44 expression are obscure in terms of the regulation of a large variety of signaling pathways although main receptor CD44 is identified as main components of CSC niche.

We next examined the connection between $\mathrm{CD} 133^{+} \mathrm{CD} 44^{-}$with vimentin and/or $\mathrm{CDH} 2$ in the cells. The results in Fig. 3(a) indicate that $\mathrm{CD} 133^{+} \mathrm{CD} 44^{-}$levels are not associated with vimentin/ $\mathrm{CDH} 2$ expression. In the $\mathrm{CD} 133^{+} \mathrm{CD} 44^{-}$level, their tan $\delta$-dependent manner is not contributed by EMT phenomenon and is an independent from acquisition of the EMT. Several studies were reported that CD133 expression contributes to tumor survival in hypoxia. Soeda et al. ${ }^{31)}$ reportedd that hypoxia could promote $\mathrm{CD} 133^{+}$ cancer stem-like cells expansion by upregulating HIF-1 $\alpha$. Hashimoto et al. ${ }^{32)}$ also showed that hypoxia could encourage CD133 expression with HIF-1 $\alpha$. However, an interaction mechanism between CD133 and HIF-1 $\alpha$ is still obscure. In the present study, we showed the possibility that CD133 does not affects HIF-1 $\alpha$ expression as well as vimentin. These findings are at odds with the hypothesis that EMT is necessary to sustain the CSC phenotype, and they imply that the expression between CD133 and CD44 markers are uncoupled each other and CD133 expression does not connect with EMT in each of the substrate conditions tested in this study.

Taken together, CSC phenotype may not be rigid. Rather, interconversion of CSCs and non-CSCs might be a relatively common phenomenon that is driven by environmental stimuli ( $\tan \delta$ feature), or simply by stochasticity.

Previous reports showed that high CD133 expression is especially correlated with tumorigenicity, metastasis, and worse prognosis ${ }^{20,33}$. It has a strong potential as a target for drug therapies, because many beast cancer cell lines have express this marker.

\section{CONCLUSIONS}

The $S$ value in MCF-7 cells was significantly upregulated with decreasing in damping coefficient $(\tan \delta)$.

For CD133 and CD44 expression of MCF-7 cells, the expression of $\mathrm{CD} 133^{-} \mathrm{CD} 44^{+}$is uncoupled from that of $\mathrm{CD} 133^{+} \mathrm{CD} 44^{-}$in MCF-7 cells under different oxygen concentration levels. The $\mathrm{CD} 133^{-} \mathrm{CD} 44^{+}$levels were promoted by damping coefficient, suggesting the softer gel substrate produce a large amount of surface molecule of CD44. For the co-expression of $\mathrm{CD} 133^{+} \mathrm{CD} 44^{+}$, seemed to be affected by the combination of both $\mathrm{CD} 133^{+} \mathrm{CD} 44^{-}$and $\mathrm{CD} 133^{-} \mathrm{CD} 44^{+}$.

EMT was more promoted in both normoxic and hypoxic environment when MCF-7 cells incubated on softer gel substrate, especially on AC-soft ( $\tan \delta$ of 0.244 ) substrate, as revealed by EMT marker genes analysis. A significant association between $\mathrm{CD} 133^{-} \mathrm{CD} 44^{+}$levels and vimentin/CDH2 expression in the cells incubated on AC-soft substrate under normoxia and hypoxia was observed, indicating the cells on softer substrate have a survival advantage due to the EMT. In contrast, for the $\mathrm{CD} 133^{+} \mathrm{CD} 44^{-}$levels, their $\tan \delta$-dependent manner was not contributed by EMT phenomenon and was an independent from acquisition of the EMT.

The substrate damping as potential physical parameter emerged the important linkage to cellular motility, cancer stemness, and EMT induction. Although further investigation is required to clarify the efficacy of environmental stimuli ( $\tan \delta$ feature) for tumors exhibiting CSC-like properties, our study indicates that the MCF-7 cells incubated on softer substrate might lead to express CSC biomarkers exhibiting high CD44 and CD133 expression. 


\section{ACKNOWLEDGMENTS}

This work was supported by the Grant in TTI as a Special Research Project (FY2017-2018).

\section{REFERENCES}

1) Health, Labour and Welfare Ministry, Japan retrieved on 16 November, 2020, from http://www.mhlw.go.jp/toukei/saikin/ hw/jinkou/geppo/nengai19/dl/h7.pdf

2) Cancer morbidity and mortality. retrieved on 16 November, 2020, from https://ganjoho.jp/reg_stat/statistics/stat/short_ pred.html

3) Allemani C, Weir HK, Carreira H, Harewood R, Spika D, Wang XS, Bannon F, Ahn JV, Johnson CJ, Bonaventure A, Marcos-Gragera R, Stiller C, Silva GA, Chen WQ, Ogunbiyi OJ, Rachet B, Soeberg MJ, You H, Matsuda T, Bielska-Lasota M, Storm H, Tucker TC, Coleman MP, Lancet, 385, 977(2015).

4) Hanahan D, Weinberg RA, Cell, 144-5, 646,(2011).

5) Craene BD, Berx G, Nat Rev Cancer, 13, 97 (2013).

6) Gil J, Stembalska A, Pesz KA, Sasjadek MM, J App Genet, 49, 193 (2008).

7) McAllister SS, Weinberg RA, Nat Cell Biol, 16, 717 (2014).

8) Taddei ML, Giannoni E, Comito G, Chiarugi P, Cancer letters, 341, 80 (2013).

9) Giordano A, Gao H, Anfossi S, Cohen E, Mego M, Lee B-N, Tin S, Laurentiis MD, Parker CA, Alvarez RH, Valero V, Ueno NT, Placido SD, Mani SA, Estava FJ, Cristofanilli M, Reuben JM, Mol Cancer Ther, 11, 2526 (2012).

10) de Souza Natalie, Nat Methods, 4, 386 (2007).

11) Yip AK, Iwasaki K, Ursekar C, Machiyama H, Saxena M, Chen H, Harada I, Chiam K-H, Sawada Y, Biophys J, 104, 19 (2013).

12) Ishikawa $Y$, Sasaki R, Domura R, Okamoto M, Mater Today Chem, 13, 8 (2019).

13) Jiang F, Khairy K, Poole K, Howard J, Müller DJ, Microsc Res Tech, 64, 435 (2004).
14) Helmuth JA, Burckhardt CJ, Greber UF, Sbalzarini IF, $J$ Struct Biol, 167,1 (2009) .

15) Tranquillo RT, Lauffenburger DA, Zigmond SH, J Cell Biol, 106-2, 303 (1988).

16) Tzvetkova-Chevolleau T, Stephanou A, Fuard D, Ohayon J, Schiavone P, Tracqui P, Biomaterials, 29, 1541 (2008).

17) O'brien CA, Pollett A, Gallinger S, Dick JE, Nature, 445, 106 (2007).

18) Yang D, Wang H, Zhang J, Li C, Lu Z, Liu J, Lin C, Li G, Qian H, Oncol Res, 21, 51 (2013).

19) Matsuda Y, Kure S, Ishiwata T, Med Mol Morphol, 45, 59 (2012).

20) Chen S, Song X, Chen Z, Li X, Li M, Liu H, Li J, PLoS ONE, 8, e56380 (2013).

21) Sahlberg SH, Spiegelberg D, Glimelius B, Stenerlöw B, Nestor M, PLoS ONE, 9, e94621 (2014).

22) Schneider M, Huber J, Hadaschik B, Siegers GM, HeinzHerbert F, Schuler J, BMC Cancer, 12, 96 (2012).

23) Xie J, Xiao Y, Zhu X-Y, Ning Z-Y, Xu H-F, Med. Oncol, 33, 42 (2016).

24) Yilmaz M, Christofori G, Cancer Metastasis Rev, 28, 15 (2009).

25) Tam WL, Weinberg RA, Nat Medicine, 19, 1438 (2013).

26) Zavadil J, Böttinger E, Oncogene, 24-37, 5764 (2005).

27) Gilkes DM, Bajpai S, Chaturvedi P, Wirtz D, Semenza GL, $J$ Biol Chem, 288-15, 10819 (2013).

28) Kreso A, Dick JE, Stem Cell, 14, 275 (2014).

29) Chaffer CL, Weinberg RA, Science, 331, 1559 (2011).

30) Giancotti FG, Cell, 155,750 (2013).

31) Soeda A, Park M, Lee D, Mintz A, Androutsellis-Theotokis A, McKay RD, Engh J, Iwama T, Kunisada T, Kassam AB, Oncogene, 28, 3949 (2009).

32) Hashimoto O, Shimizu K, Semba S, Chiba S, Ku Y, Yokozaki H, Hori Y, Pathobiology, 78, 181 (2010).

33) Sahlberg SH, Spiegelberg D, Glimelius B, Stenerlöw B, Nestor M, PLoS ONE, 9, e94621 (2014). 